\title{
Preparation of Photoluminescent Microporous Hybrid Films by Breath Figure Method
}

\author{
Hai-yan Chen ${ }^{1,2}$, Ting Zhu ${ }^{1}$, Wei-chao Xu ${ }^{1}$, Ming Zhang ${ }^{1,3 *}$ \\ (1. School of Chemistry and Chemical Engineering, Yangzhou University, Jiangsu Yangzhou, Zip Code 225002,China \\ 2. Yangzhou Polytechnic College, Jiangsu Yangzhou, Zip Code 225127, China \\ 3. Yangzhou University test center, Jiangsu Yangzhou, Zip Code 225009,China) \\ *Corresponding Author: Ming Zhang,lxyzhangm@yzu.edu.cn
}

\begin{abstract}
Photoluminescent microporous hybrid films with honeycomb patterns were prepared by Breath Figure method via a straightforward, one-step process by doping complex containing $\mathrm{Eu}$ into polystyrene-b-polyacrylic (PS-b-PAA) solution. Several key influencing factors, such as the concentration of the block polymer solution, the relative humidity of the atmosphere and the substrates, were investigated to control micro pore size and tune film surface properties. Meanwhile the optical properties of the obtained micro porous hybrid films were analyzed by the ultraviolet and emission spectra.
\end{abstract}

Keywords: microporous hybrid film, breath figure method, photoluminescent, PS-b-PAA block copolymer.

\section{Introduction}

Recently, highly ordered polymer films have sparked a wide interest due to their potential application in numerous fields such as electronics, optics, and biotechnology ${ }^{[1-3]}$. Among those techniques for the fabrication of microporous structured films, the breath figure method is based on evaporative cooling and subsequent water-droplet templating to form an ordered array of breath figures and proved to be an effective dynamic template method due to its facility, economy, large area applicability and robust mechanism of pattern formation ${ }^{[4-6]}$.

Amphiphilic block copolymer is generally considered to be a kind of ideal material for preparing ordered porous film, for amphiphilic block copolymer in the selective solvent has a high segment density and can be gathered in the interface layer of organic solvent and water so as to effectively stabilize the water droplets, preventing the mutual cohesion between the water droplets.

In this work, rare earth complex was first introduced to the breath figure method as an excellent candidate for functional materials, due to their unique electronic configuration, physical and chemical properties. By doping complex containing $\mathrm{Eu}$ into polystyrene-b-polyacrylic (PS-b-PAA) solution, photoluminescent microporous hybrid films with honeycomb patterns were prepared easily under high relative humidity.

The present research draws a convenient and effective route to fabricate fluorescent honeycomb films, and establishes the multicomponent self-assembly in honeycomb films to endow the films with increased functionality, revealing potential applications in sensor, micro-reactor and catalysis.

\section{Experimental Section}

\subsection{Materials}

Rare earth oxides were purchased from Liyang Rare Earth Company. Acrylic acid, styrene, tetrahydrofuran (THF)and chloroform $\left(\mathrm{CHCl}_{3}\right)$ were purchased from Shanghai Chemical Reagent Plant. All reagents were of analytical grade and were used without further purification.

The preparation of amphiphilic diblock copolymer, poly-styrene-b-poly(acrylic acid) (PS-b-PAA), was synthesized via atom-transfer radical polymerization with a similar procedure as reported in ref 7.

Synthesis of rare earth complex was conducted by sedimentation and direct method as reported in ref 8 . 


\subsection{Preparation of the microporous hybrid Films}

The glass substrates were cleaned by acetone and air dried. Polymer and rare earth complex were mixed with a fixed weight ratio and dissolved in $\mathrm{CHCl}_{3}$. The solution concentration was $10 \mathrm{mg} \mathrm{mL}^{-1}$. The honeycomb film was prepared by casting $10 \mu \mathrm{L}$ of solution onto the substrate with a microsyringe under high relative humidity. With organic solvent volatilization, the transparent solution became turbid. The film was taken out for microscope observation after complete solvent evaporation. All the experiments were carried out at room temperature unless stated otherwise.

\subsection{Characterization and apparatus}

Scanning electronic microscopy (SEM) images were obtained using a Hitachi S4800 scanning electron microscope. A $10 \mathrm{keV}$ electron beam was used for the observation with a working distance of $10 \mathrm{~mm}$ in order to obtain secondary.

The excitation and emission spectra were detected by using the fluorescent spectrometer, model FLS920.

\section{Results and discussion}

\subsection{Influence of humidity and solution concentration}

During breath figure progress to prepare highly ordered porous polymer membranes, the environmental conditions, such as ambient temperature, relative humidity, properties of solvent, spreading volume and substrates, have serious impact on the solvent evaporation and affect the microstructure and surface morphology of membranes produced $^{[9-11]}$.

It is generally considered that humid atmosphere, which could be provided by static humid atmosphere or controlled by adjusting the air flow rate, is an essential parameter for breath figure method. The use of humid airflow instead of static conditions could make solvent evaporation easier and allow BFs generation even for solvents with a relatively high boiling point ${ }^{[12]}$. Thus we take the dynamic figure breathing method to control high relative humidity by adjusting the air flow rate. Fig. 1 shows the dependence of the pore size on relative humidity. It can be clearly seen that the pore size increased from nano-meter (Fig.4a) to micro-meter (Fig.4c) with an increase of relative humidity from $55 \%$ to $75 \%$. This can be attributed to the fact that the pore size and periodicity of a polymer film is determined by the size and volume of the water droplets which are increased with relative humidity. It is interesting to notice that when relative humidity is under $50 \%$ there was only transparent film and no pores were observed, while when relative humidity is above $85 \%$, large disordered pores were observed (Fig.4d), which verified that the water droplet array as a template is a key parameter

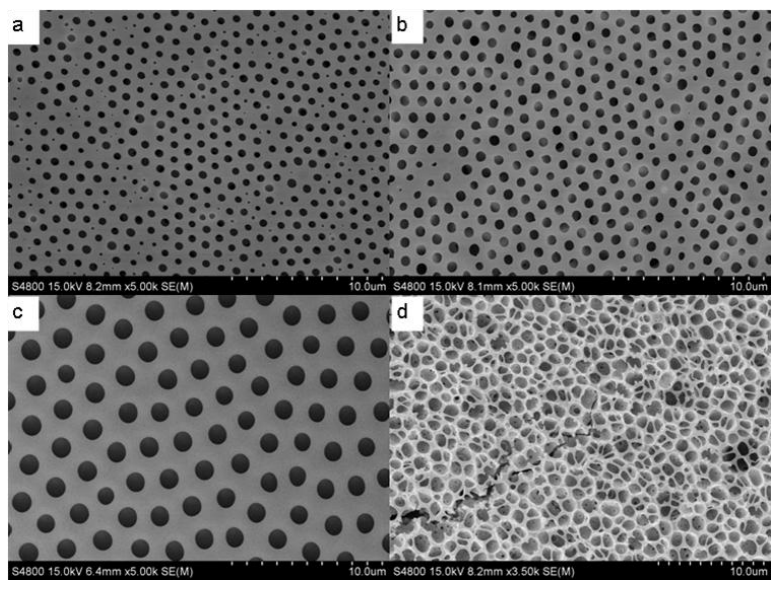

Fig.1 SEM images of the porous films at different humidity (a) $55 \%$, (b) $65 \%$, (c) $75 \%$, (d) $>85 \%$. Other conditions: temperature: $25^{\circ} \mathrm{C}$, humidity: $75 \%$, spreading volume: $10 \mu 1$.

for BF formation.

The effects of the amount of rare earth complexes on final porous hybrid membrane morphology were also investigated. Fig.2 shows SEM images of the porous hybrid films with different amount of rare earth complexes. As shown in Fig.2, it can be clearly seen that with increasing the amount of rare earth complexes from 0.1 $\mathrm{mg} / \mathrm{mL}$ to $1 \mathrm{mg} / \mathrm{mL}$, size and the size distribution of the holes decrease markedly, together with the arrangement of the holes becomes disordered gradually, indicating the effective inhibition of rare earth complexes for the coagulation of water droplets.

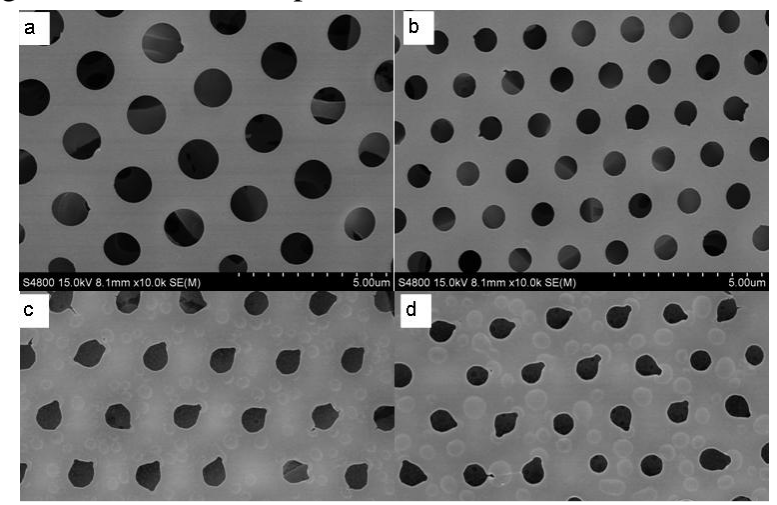

Fig. 2 SEM images of the porous films at different concentration of complexes (a)0.1, (b)0.25; (c)0.5; (d) $1 \mathrm{mg} / \mathrm{ml}$. Other conditions: temperature: $25^{\circ} \mathrm{C}$, humidity: $75 \%$, spreading volume: $10 \mu \mathrm{l}$. 


\subsection{Uv absorption spectra and fluorescence properties of porous hybrid film}

In order to assess optical properties of the porous hybrid film, Uv absorption spectra and fluorescence properties were analyzed. As shown in Fig.3, it can be clearly seen that the porous hybrid film with rare earth complexes shows characteristics absorption peak of europium at $325 \mathrm{~nm}$. When the porous hybrid film was irradiated with $254 \mathrm{~nm}$, it showed a strong red fluorescence emission, which indicated that the good photoluminescence performance was well maintained.
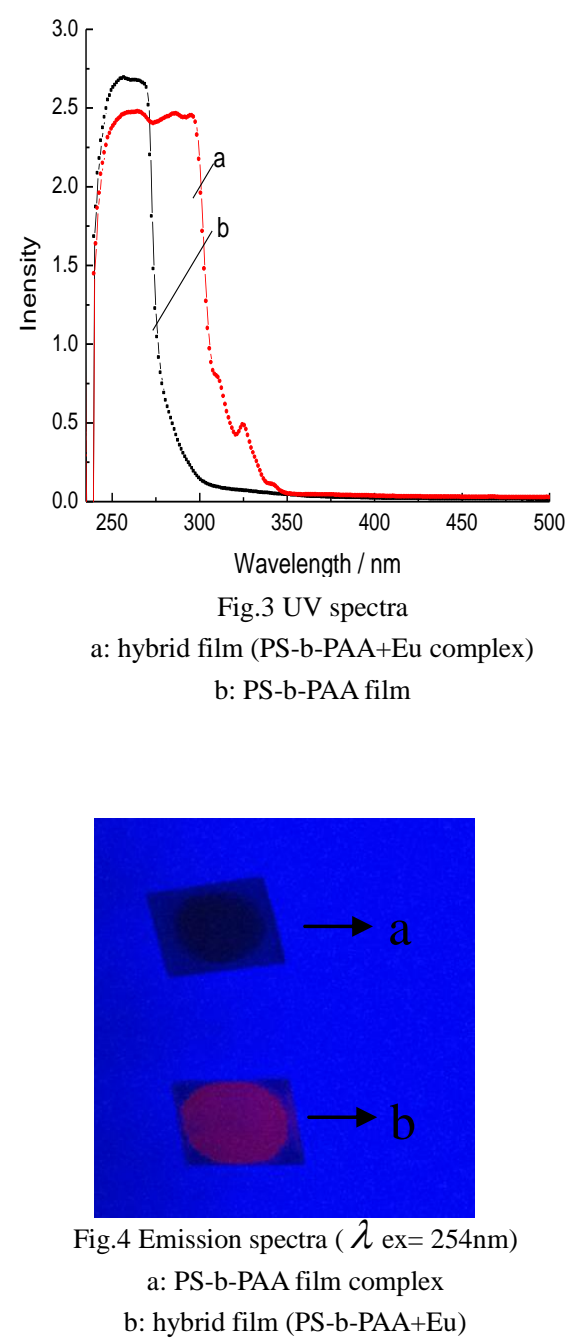

\section{Conclusions}

Photoluminescent microporous hybrid films were prepared by Breath Figure method via a straightforward, one-step process by doping complex containing $\mathrm{Eu}$ into polystyrene-b-polyacrylic (PS-b-PAA) solution. It can be induced from the ultraviolet spectra that the chemical structure and physical properties of complex containing Eu are well maintained in micro porous hybrid film.

The present research draws a convenient and effective route to fabricate fluorescent honeycomb films, and establishes the multicomponent self-assembly in honeycomb films to endow the films with increased functionality, revealing potential applications in sensor, micro-reactor and catalysis.

\section{Acknowledgments}

This work was supported by the Foundation of Key Laboratory of Environmental materials \& engineering, Jiangsu, China (K13073).

\section{References}

(1) Liang C, Hong K, Guiochon G A, et al. Synthesis of a Large-Scale Highly Ordered Porous Carbon Film by Self - Assembly of Block Copolymers [J]. Angewandte Chemie International Edition, 2004, 43(43): 5785-5789.

(2) Tang C, Lennon E M, Fredrickson G H, et al. Evolution of block copolymer lithography to highly ordered square arrays [J]. Science, 2008, 322(5900): 429-432.

(3) Umeda T, Tokito S, Kumaki D. High-mobility and air-stable organic thin-film transistors with highly ordered semiconducting polymer films [J]. Journal of Applied Physics, 2007, 101(5): 054517.

(4) Chen J, Yan X, Zhao Q, et al. Adjustable supramolecular polymer microstructures fabricated by the breath figure method [J]. Polymer Chemistry, 2012, 3(2): 458-462.

(5) Li J, Zhao Q L, Chen J Z, et al. Highly ordered microporous films containing a polyolefin segment fabricated by the breath-figure method using well-defined polymethylene-b-polystyrene copolymers [J]. Polymer Chemistry, 2010, 1(2): 164-167.

(6) Cong H, Wang J, Yu B, et al. Preparation of a highly permeable ordered porous microfiltration membrane of brominated poly (phenylene oxide) on an ice substrate by the breath figure method [J]. Soft Matter, 2012, 8(34): 8835-8839.

(7) ZJ Zhang. ATRP Synthesis of PS-b-PtBMA and Preparation of Porous film [D]. Yangzhou: Yangzhou 
University, 2012

(8) $\mathrm{CH}$ Yan, TW Wang, M Zhang, et al. Co-polymerization of rare earth carboxylate complex monomers [J]. Chinese Journal of Rare Earths, 2003, 21(2): 170-173.

(9) Ferrari E, Fabbri P, Pilati F. Solvent and substrate contributions to the formation of breath figure patterns in polystyrene films [J]. Langmuir, 2011, 27(5): 1874-1881.

(10) Li L, Zhong Y, Li J, et al. Breath figure lithography: A facile and versatile method for micropatterning [J]. Journal of colloid and interface science, 2010, 342(1): 192-197.

(11) Wan L S, Li J W, Ke B B, et al. Ordered microporous membranes templated by breath figures for size-selective separation [J]. Journal of the American Chemical Society, 2011, 134(1): 95-98.

(12) Lei $\mathrm{Li}$, Yawen Zhong, Chunyin Ma, et al. et al. Honeycomb-Patterned hybrid films and their template applications via a tunable amphiphilic block polymer/inorganic precursor system [J]. Journal of Chem. Mater. 2009, 21, 4977-4983 4977 